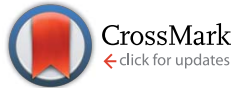

Cite this: RSC Adv., 2015, 5, 77746

Received 28th July 2015

Accepted 9th September 2015

DOI: $10.1039 / c 5 r a 14970 c$

www.rsc.org/advances

\title{
Liposome collapse resulting from an allosteric interaction between 2,6-dimethyl- $\beta$-cyclodextrins and lipids $\uparrow$
}

\author{
Atsushi Ikeda, ${ }^{* a}$ Noboru Iwata, ${ }^{a}$ Shodai Hino, ${ }^{a}$ Tomoya Mae, ${ }^{a}$ Yuki Tsuchiya, \\ Kouta Sugikawa, ${ }^{a}$ Takehiro Hirao, ${ }^{\text {b }}$ Takeharu Haino, ${ }^{b}$ Kazuaki Oharac \\ and Kentaro Yamaguchic
}

Although heptakis(2,6-di-O-methyl)- $\beta$-cyclodextrin (DMe- $\beta$-CDx) has been reported to exhibit higher cytotoxicity than many other cyclodextrins because of the way in which it abstracts cholesterols from liposomes, we have identified another reason for its cytotoxicity based on its interaction with lipids. These interactions exhibited nonlinear sigmoidal responses with Hill coefficient values $(n)$ in the range of 3.0-3.6, which indicated that this phenomenon involves positive allosterism. Furthermore, analysis by mass spectroscopy revealed that the lipid-DMe- $\beta$-CDx complexes had stoichiometric ratios in the range of $1: 1-1: 4$.

\section{Introduction}

Cyclodextrins (CDxs) and their derivatives are water-soluble host molecules with a hydrophobic cavity. ${ }^{1}$ Considerable research has been directed towards the use of CDxs as drug carriers during the last two decades because these compounds can form inclusion complexes with hydrophobic drugs that possess improved aqueous solubility and stability properties compared with the parent drugs. $^{2-4}$ The toxicity of CDxs is one of their most important characteristics and plays a significant role in defining their overall utility, as well as the scope of their potential applications. ${ }^{5,6}$ It has been reported that natural CDxs, such as $\alpha$-CDx and $\gamma$-CDx, are essentially non-toxic towards viruses, bacteria and animal cells. ${ }^{7,8}$ In contrast, some chemically modified CDxs have been shown to exhibit high toxicity, including heptakis(2,6-di-O-methyl)- $\beta$-cyclodextrin (DMe- $\beta$-CDx). ${ }^{7,8}$ The toxicities of several known CDxs and there derivatives follow the general trend DMe- $\beta$-CDx $\gg \beta$-CDx $>$ heptakis(2,3,6-tri-O-methyl)- $\beta$-cyclodextrin (TMe- $\beta$-CDx) $\approx \alpha$-CDx $>\gamma$-CDx. The high toxicity of DMe- $\beta$-CDx has been attributed not only to its ability to extract cholesterol ${ }^{9,10}$ but also to its interaction with lipids. ${ }^{11-13}$ For example, Nishijo et al. reported that the interaction of DMe- $\beta$-CDx with liposomes had a significant impact

\footnotetext{
${ }^{a}$ Department of Applied Chemistry, Graduate School of Engineering, Hiroshima University 1-4-1 Kagamiyama, Higashi-Hiroshima 739-8527, Japan. E-mail: aikeda@hiroshima-u.ac.jp

${ }^{b}$ Department of Chemistry, Graduate School of Science, Hiroshima University, 1-3-1 Kagamiyama, Higashi-Hiroshima 739-8526, Japan

${ }^{c}$ Faculty of Pharmaceutical Sciences at Kagawa Campus, Tokushima Bunri University 1314-1 Shido, Sanuki, Kagawa 769-2193, Japan

$\dagger$ Electronic supplementary information (ESI) available: Average hydrodynamic diameter values and UV-vis absorption, ${ }^{1} \mathrm{H}$ NMR, NOESY and CSI-MS spectra. See DOI: $10.1039 / \mathrm{c} 5 \mathrm{ra} 14970 \mathrm{c}$
}

on the phase transition temperature of the liposomes, as well as causing a change in the enthalpy during the liposomal phase transition, which was detected by differential scanning calorimetry. ${ }^{12}$ Furthermore, Piel et al. confirmed that DMe- $\beta$-CDx can be used to extract lipids from liposomes based on the results of an experiment involving the release of calcein from liposomes. ${ }^{13}$ Both of these papers also reported that DMe- $\beta$-CDx perturbed the integrity of the lipid membranes of liposomes. In this study, we have investigated the DMe- $\beta$-CDx-mediated collapse of liposomes and identified a new mechanism for the perturbation and extraction of lipids from liposomes, involving the formation of lipid-DMe- $\beta$-CDx complexes.

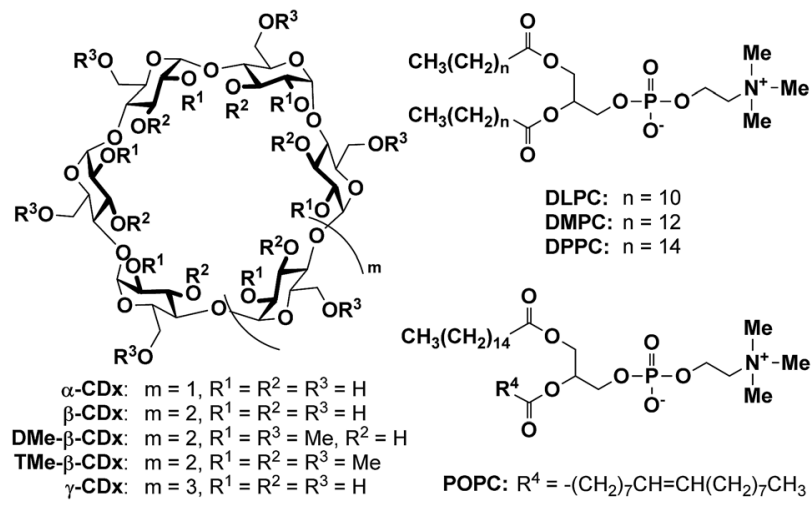

\section{Results and discussion}

Effect of CDxs towards the stability of liposomes

The morphological changes in the liposomes were evaluated by UV-vis absorption spectroscopy based on variations in their 
light scattering intensity at $450 \mathrm{~nm}$ (Fig. $1 \mathrm{~A}$ and $\mathrm{S} 1 \dagger$ ). The light scattering intensity $\left(A / A_{0}\right.$ at $\left.450 \mathrm{~nm}\right)$ of a 1,2-dimyristoyl-snglycero-3-phosphocholine (DMPC)-based liposome decreased following the addition of DMe- $\beta$-CDx (Fig. 1A). This behaviour is similar to that observed for the collapse of liposomes following the addition of a suitable lysing agent (e.g., ethanol) or a surfactant under aqueous conditions. ${ }^{\mathbf{1 4 , 1 5}}$ As shown in Fig. 1A, the addition of more than 10 equivalents of DMe- $\beta$-CDx led to the collapse of the DMPC liposomes because the $A / A_{0}$ value went below 0.1 . The $A / A_{0}$ value also decreased following the addition of $\alpha$-CDx (Fig. 1A) and gave a very similar curve to that of DMe- $\beta$ CDx. However, in contrast to the clear solution obtained after the addition of DMe- $\beta$-CDx, a finely dispersed precipitate was observed immediately after the mixing of $\alpha$-CDx with the DMPC-liposome solution. It is noteworthy that Csempesz et al. reported the same phenomenon for the mixing of 1,2-dipalmitoyl-sn-glycero-3-phosphocholine (DPPC) and $\alpha$-CDx. ${ }^{16}$ It can be difficult to analyse the nature of the interactions between DMPC and $\alpha$-CDx because the resulting precipitate is insoluble in water. Furthermore, the aqueous solubility of $\beta$-CDx was too low to measure the intensity of its light scattering at $450 \mathrm{~nm}$ by UVvis absorption spectroscopy under the same conditions ([ $\beta$ $\mathrm{CDx}] /[\mathrm{DLPC}]=0-5$ equiv.) (Fig. $1 \mathrm{~A})$. Until $[\beta-\mathrm{CDx}] /[\mathrm{DLPC}]=5$ equiv., the addition of $\beta$-CDx had very little impact on the light scattering intensity of the liposome (Fig. 1A), and it became increasingly difficult to add more $\beta$-CDx to the mixture. For these reasons, $\alpha$-CDx and $\beta$-CDx were not taken forward into the
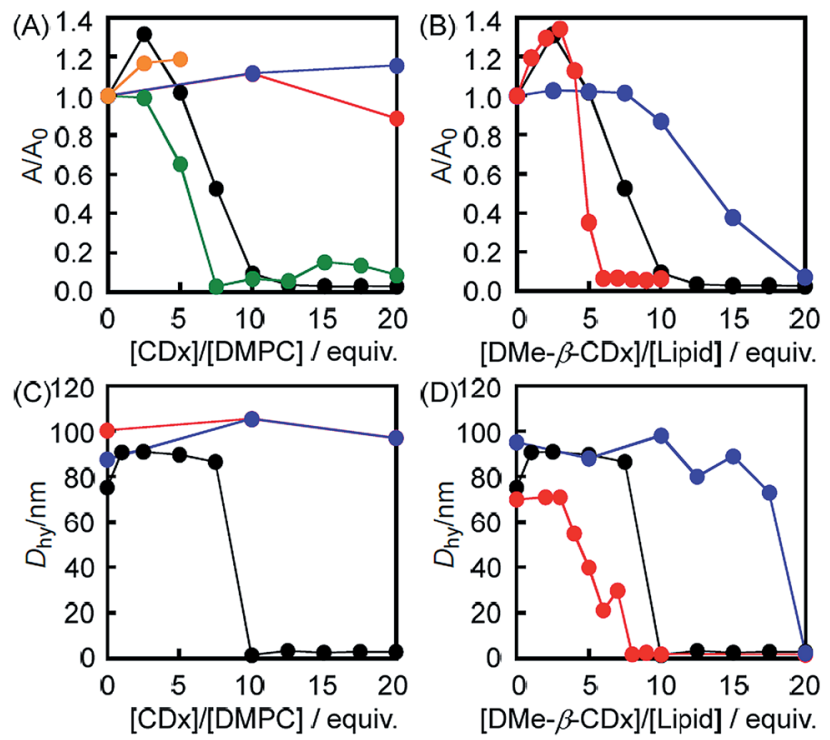

Fig. 1 Changes in the intensity $\left(A / A_{0}\right)$ of the light scattering at $450 \mathrm{~nm}$ by UV-vis absorption spectroscopy for $(A)$ the DMPC liposomes before and after the addition of DMe- $\beta-C D x$ (black), $\alpha-C D x$ (green), $\beta-C D x$ (orange), TMe- $\beta-C D x$ (red) and $\gamma-C D x$ (blue) ([DMPC] $=1.0 \mathrm{mM}$ ) and (B) the DMPC (black), DLPC (red) and DPPC (blue) liposomes before and after the addition of DMe- $\beta-C D x$ ([lipids] $=1.0 \mathrm{mM}$ ). Hydrodynamic diameters $\left(D_{\text {hy }}\right)$ of $(C)$ the DMPC-liposome before and after the addition of DMe- $\beta$-CDx (black), TMe- $\beta$-CDx (red) and $\gamma$-CDx (blue) $\{[C D x] /[D M P C]=10(\mathrm{~mol} / \mathrm{mol})\}$ and $(\mathrm{D})$ the DMPC (black), DLPC (red) and DPPC (blue) liposomes before and after the addition of DMe- $\beta$ $\mathrm{CDx}$ ([lipids] $=1.0 \mathrm{mM}$ ). subsequent experiments. The addition of TMe- $\beta$-CDx and $\gamma$-CDx had very little impact on the light scattering intensity of the liposome (Fig. 1A), ${ }^{17}$ which indicated that these compounds did not have an adverse impact on the stability of the DMPC liposomes, even at high concentrations. The reasons for these results will be discussed in greater detail below. Taken together, however, these results indicate that the collapse of the liposomes was specific to DMe- $\beta$-CDx.

These conclusions were further supported by the average hydrodynamic diameters $\left(D_{\text {hy }}\right)$, which were determined by dynamic light scattering (DLS) on a spectrophotometer (Fig. 1C). The results of this analysis revealed that the average $D_{\text {hy }}$ value dropped sharply following the addition of 10 equiv. of DMe- $\beta$-CDx, which was indicative of the collapse of the liposomes. The $D_{\text {hy }}$ value therefore provided a clear suggestion that the DMPC-DMe- $\beta$-CDx complex did not form small aggregates in water such as micelles (5-10 $\mathrm{nm})$ but that it simply dissolved in an isolated state (Table S1, $\uparrow[\mathrm{DMe}-\beta-\mathrm{CDx}] /[\mathrm{DMPC}]=10-20$ equiv.: $\left.D_{\text {hy }}=1.6-3.4 \mathrm{~nm}\right)$. In contrast, the average $D_{\text {hy }}$ value remained constant in the presence of excess amounts of TMe- $\beta$ $\mathrm{CDx}$ and $\gamma$-CDx $([\mathrm{CDx}] /[\mathrm{DMPC}]=10$ equiv.). These results were therefore consistent with those for the intensity of light scattering at $450 \mathrm{~nm}$ by UV-vis absorption spectroscopy.

\section{Effect of lipids toward stabilities of liposomes}

1,2-Dilauroyl-sn-glycero-3-phosphocholine (DLPC: $n=10$ ) and 1,2-dipalmitoyl-sn-glycero-3-phosphocholine (DPPC: $n=14$ ) were used to investigate the effects of the alkyl chain length of the lipid on the light scattering intensity $\left(A / A_{0}\right)$ instead of DMPC $(n=12)$ (Fig. 1B and $\mathrm{S} 3 \dagger$ ). The results showed that there was a significant decrease in the light scattering intensity $\left(A / A_{0}\right)$ of the DLPC liposomes following the addition of a small amount of DMe- $\beta$-CDx $([\mathrm{CDx}] /[\mathrm{DLPC}]=6$ equiv. $)$ compared with the DMPC liposomes (10 equiv.) (Fig. 1B). In contrast, the light scattering intensity of the DPPC liposome changed only slightly following the addition of DMe- $\beta-\mathrm{CDx}([\mathrm{CDx}] /[\mathrm{DPPC}]=10$ equiv. $)$ when the mixture was heated at $30{ }^{\circ} \mathrm{C}$. However, the light scattering intensity $\left(A / A_{0}\right)$ of this mixture eventually became less than 0.1 following the addition of a larger amount of DMe- $\beta$-CDx ([CDx]/ $[\mathrm{DPPC}]=20$ equiv.). These results clearly showed that the stability of the different liposomes towards DMe- $\beta$-CDx was of the order DPPC $>$ DMPC $>$ DLPC which reflected the increasing order of the alkyl chain length. The reason for this difference will be discussed in greater detail below.

The size distributions of the different liposomes were measured by DLS both before and after addition of 10 equiv. of DMe- $\beta$-CDx. Fig. 1D shows the average $D_{\text {hy }}$ values for all of the liposomes evaluated in the current study. When the average $D_{\text {hy }}$ value became less than $5 \mathrm{~nm}$ (Fig. $1 \mathrm{D}$ and Table S1†), the minimum addition of DMe- $\beta$-CDx led to a decrease the size of the liposomes of the order DPPC > DMPC > DLPC. Although these results were effectively consistent with those reported above for the changes in the intensity of the light scattering, the slopes of the average $D_{\text {hy }}$ values became steeper following the addition of DMe- $\beta$-CDx. For example, in the [DMe- $\beta$-CDx]/ $[\mathrm{DMPC}]=7.5$ equiv. and $[\mathrm{DMe}-\beta-\mathrm{CDx}] /[\mathrm{DPPC}]=17.5$ equiv. 
cases, the intensities of the light scattering were reduced to roughly one-half of their original values (Fig. 1B), whereas the average $D_{\text {hy }}$ values remained constant at 90-100 nm (Fig. 1D). These results therefore suggested that the removal of some of the lipids from the liposomes by DMe- $\beta$-CDxs led to the formation of small liposomes, which fused with each other to

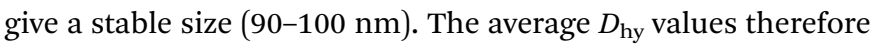
remained constant and the reduction in the intensities of the light scattering could be attributed to the decrease in the number of liposomes.

\section{Visual examination of the liposome solutions}

It is noteworthy that all of the changes described above could be observed by visual examination. As shown in Fig. 2A, an aqueous solution of the DMPC liposomes had a similar turbidity to that of soapy water. The addition of DMe- $\beta$-CDx led to the clarification of these turbid solutions (Fig. 2B and C). However, the turbidity of these solutions did not change following the addition of TMe- $\beta$-CDx or $\gamma$-CDx ([CDx] $][$ DMPC $]=20$ equiv.). The addition of DMe- $\beta$-CDx to a solution of the DMPC or DLPC liposomes ([DMe- $\beta-\mathrm{CDx}] /[\mathrm{lipid}]=10$ equiv.) led to an increase in the clarity of both solutions, with the DLPC solution becoming completely clear (Fig. 2B and G). In contrast, the addition of 10 equiv. of DMe- $\beta$-CDx to a turbid solution of DPPC liposomes had very little impact on the turbidity of the solution (Fig. 2J), with the solution only becoming clear following the addition of at least 20 equiv. of DMe- $\beta$-CDx (Fig. 2K). These results were therefore consistent with those for the changes in the light scattering and $D_{\text {hy }}$ values, as shown in Fig. 1.

\section{Morphological change of giant liposome by the addition of CDx}

Giant unilamellar vesicles (GUVs) were used in this study because they are comparable in size to cells and can be observed

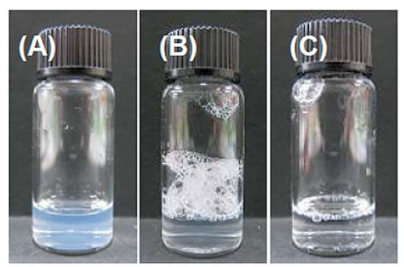

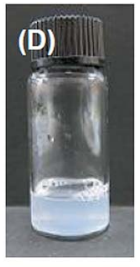

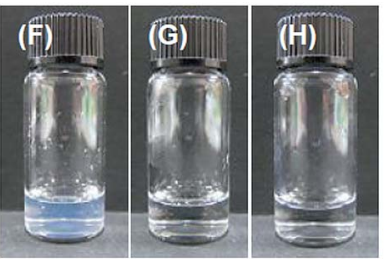

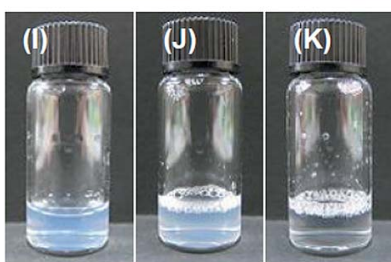

Fig. 2 Photographic images of aqueous solutions of the DMPC liposomes both (A) before and after the addition of (B) 10 equiv. and (C) 20 equiv. of DMe- $\beta-C D x$. Photographic images of the DMPC liposomes after the addition of (D) 20 equiv. of TMe- $\beta-C D x$ and (E) 20 equiv. of $\gamma$ CDx. Photographic images of the DLPC liposomes (F) before and after the addition of $(G) 10$ equiv. and $(H) 20$ equiv. of DMe- $\beta$-CDx. Photographic images of the DPPC liposomes (I) before and after the addition of (J) 10 equiv. and (K) 20 equiv. of DMe- $\beta$-CDx ([lipids] $=1.0 \mathrm{mM}$ ).

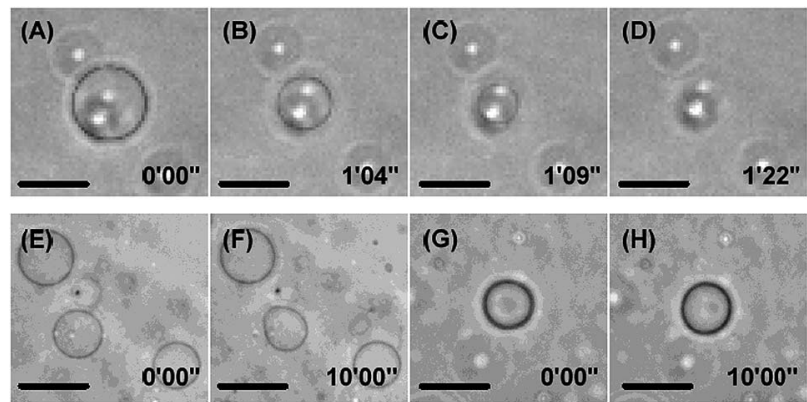

Fig. 3 Time-lapse images of GUVs consisting of POPC following the addition of (A)-(D) DMe- $\beta-C D x$, (E and F) TMe- $\beta-C D x$ and ( $G$ and $H$ ) $\gamma$-CDx solutions at ambient temperature $([C D x]=10.0 \mathrm{mM})$. All of the micrographs were recorded as phase contrast images. The time elapsed following the starting injection of the CDx solution through the micropipette has been indicated for each image. All of the scale bars show $10 \mu \mathrm{m}$

directly by optical microscopy. Aqueous solutions of DMe- $\beta$ CDx, TMe- $\beta$-CDx and $\gamma$-CDx $(20 \mathrm{mM})$ were added to GUVs consisting of 1-palmitoyl-2-oleoyl-sn-glycero-3-phosphatidylcholine (POPC), and time-lapse contrast images of the resulting mixtures were obtained by confocal laser microscopy (Fig. 3 and S4 $\dagger)$. The results for the addition of DMe- $\beta$-CDx revealed that the GUVs started to shrink after a period of $1 \mathrm{~min}$ (Fig. 3B and C) and that they had disappeared completely after $1 \mathrm{~min} 22 \mathrm{~s}$ (Fig. 3D).$^{18}$ To confirm that the observed shrinkage of the GUVs could be attributed to changes in the osmotic pressure resulting from the addition of a large excess of DMe- $\beta-C D x$ we also investigated the addition profiles of TMe- $\beta$-CDx and $\gamma$-CDx under the same conditions. The results for these two CDxs revealed that there were no changes in the shape of the GUVs, which indicated that the addition of a large excess of TMe- $\beta$-CDx or $\gamma$-CDx had no discernible impact on the morphology of the lipid membrane (Fig. $3 \mathrm{~F}$ and $\mathrm{H}$ ).

\section{Structure determination of lipid-CDx complexes by ${ }^{1} \mathrm{H}$ NMR analyses}

The formation of the lipid-CDx complexes was confirmed by ${ }^{1} \mathrm{H}$ NMR analysis. In the absence of a CDx derivative, the ${ }^{1} \mathrm{H}$ NMR spectrum of the DMPC liposomes did not contain any peaks that could be assigned to DMPC because of the extreme broadening of the signals resulting from the formation of the liposomes (Fig. 4A). However, several new peaks emerged in the range of $0.8-1.4 \mathrm{ppm}$ following the addition of DMe- $\beta-\mathrm{CDx}$, which were assigned to the alkyl chains of the individual DMPC molecules because they were very similar to those observed in the ${ }^{1} \mathrm{H}$ NMR spectrum of DMPC dispersed in chloroform (Fig. $4 \mathrm{~B}$ and $\mathrm{S} 5 \dagger$ ). This result therefore suggested that the addition of DMe- $\beta$-CDx led to the collapse of the DMPC liposomes and that the isolation of the DMPC-DMe- $\beta$-CDx complex in water was in consistent with the results reported above for the changes in the light scattering intensity (Fig. 1A). The signals for free DMe- $\beta$-CDx and those of its complex with DMPC did not appear separately but merely shifted, which indicated that the rate of the complexation-decomplexation exchange of 

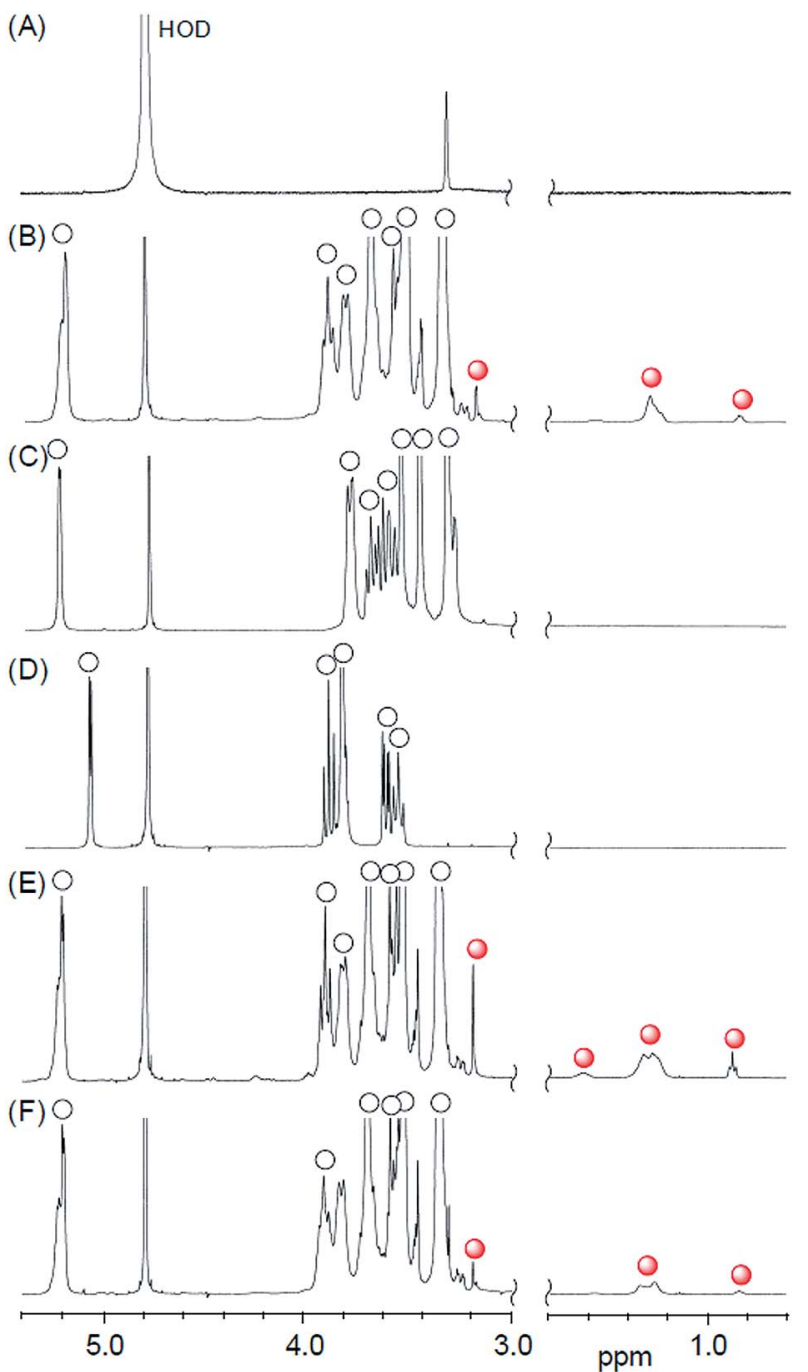

Fig. 4 Partial ${ }^{1} \mathrm{H}$ NMR spectra of (A) DMPC liposome, (B) DMPC-DMe$\beta$-CDx complex, (C) DMPC-TMe- $\beta$-CDx complex, (D) DMPC- $\gamma-C D x$ complex, (E) DLPC-DMe- $\beta-C D x$ complex and (F) DPPC-DMe- $\beta-C D x$ complex, $\{$ llipid] $=1.0 \mathrm{mM},[C D x] /[$ lipid] $=20$ equiv., open circles: $C D x$, red circles: lipid\}. $\mathrm{D}_{2} \mathrm{O}, 23^{\circ} \mathrm{C}, 400 \mathrm{MHz}$.

DMe- $\beta$-CDx was occurring too quickly to be observed on the ${ }^{1} \mathrm{H}$ NMR time scale. In contrast, the addition of TMe- $\beta$-CDx or $\gamma$-CDx to a solution of DMPC liposomes did not lead to the appearance of any new peaks in the range of 0.8-1.4 ppm (Fig. 4C and D), which indicated that these CDxs could not interact with DMPC at the same concentration $([\mathrm{DMPC}]=1.0$ $\mathrm{mM},[\mathrm{CDx}] /[\mathrm{DMPC}]=20$ equiv.). The association constants of the DMPC-TMe- $\beta$-CDx and $\gamma$-CDx complexes were therefore much lower than that of the DMPC-DMe- $\beta$-CDx complex. These results were therefore in agreement with those reported above for the slight changes in the light scattering intensity of the lipids following the addition of TMe- $\beta$-CDx and $\gamma$-CDx.

As shown in Fig. 4E and F, S6 and $\mathrm{S} 7, \uparrow$ new peaks appeared in the ${ }^{1} \mathrm{H}$ NMR spectra of the DLPC and DPPC liposomes in the range of 0.8-1.4 ppm, which were assignable to the alkyl chains of individual molecules of DLPC and DPPC, respectively. These results therefore indicated that the DLPC and DPPC liposomes collapsed to form the corresponding DMe- $\beta$-CDx complexes following the addition of DMe- $\beta$-CDx $([\mathrm{DMe}-\beta-\mathrm{CDx}] /[$ lipid $]=$ 20 equiv.).

The geometry of the DMPC-DMe- $\beta$-CDx complex was confirmed by a $2-\mathrm{D}^{1} \mathrm{H}^{-1} \mathrm{H}$ NOESY experiment. NOE cross-peaks were observed for the DMPC-DMe- $\beta$-CDx complex between the alkyl chains of the DMPC molecules (0.9 and 1.2-1.4 ppm) and the protons of DMe- $\beta$-CDx (Fig. 5 and S8 $\dagger$ ). Strong cross-peaks were also observed for the $\mathrm{H}-3, \mathrm{H}-5, \mathrm{H}-6$ and $\mathrm{C}-6-\mathrm{CH}_{3}$ protons of DMPC, which were positioned inside of the CDx cavity (Fig. 5). No cross-peaks were observed for the $\mathrm{H}-4$ proton, which indicated that this proton was outside of the CDx cavity. Furthermore, no cross-peaks were observed between the N-Me moieties on the head group of DMPC and any of the protons of DMe- $\beta$-CDx. These results therefore confirmed that DMe- $\beta$-CDx had encapsulated the alkyl chains of DMPC inside its cavity and that the resulting complex had a pseudorotaxane conformation (Scheme 1).

These ${ }^{1} \mathrm{H}$ NMR data indicated that TMe- $\beta$-CDx and $\gamma$-CDx could not form a pseudorotaxane conformation with the lipids. It has been reported that $\gamma$-CDx can encapsulate poly(methylvinylether) ${ }^{19}$ and polyisobutylene ${ }^{20}$ in its cavity but not poly(ethylene glycol). These results therefore suggest that the cavity of $\gamma$-CDx is too large to adequately accommodate the alkyl chains of the lipids. Furthermore, $\gamma$-CDx cannot form inclusion complexes with lipids via an induced-fit mechanism because of

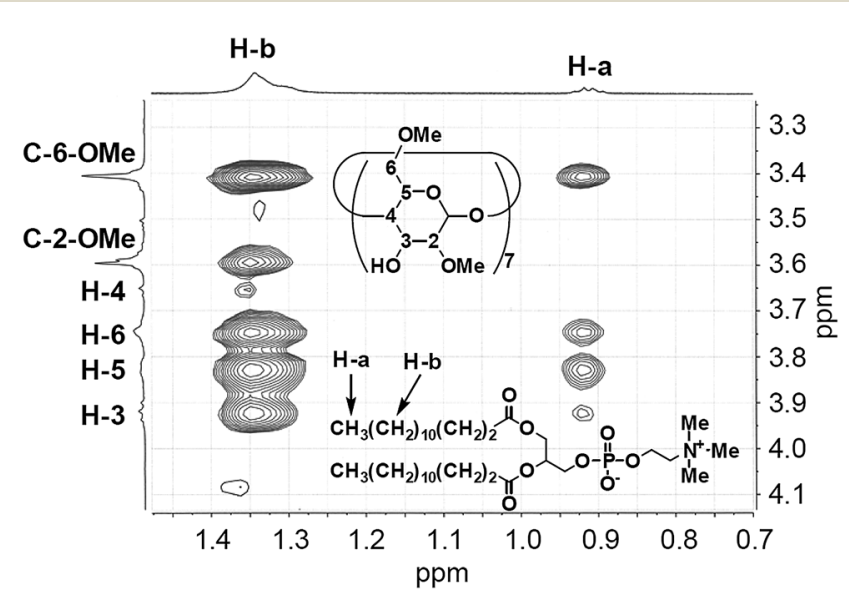

Fig. 5 Expansion of the cross-peaks between DMPC and DMe- $\beta-C D x$ in the NOESY spectrum of the DMPC-DMe- $\beta$-CDx complex (Fig. S8广).

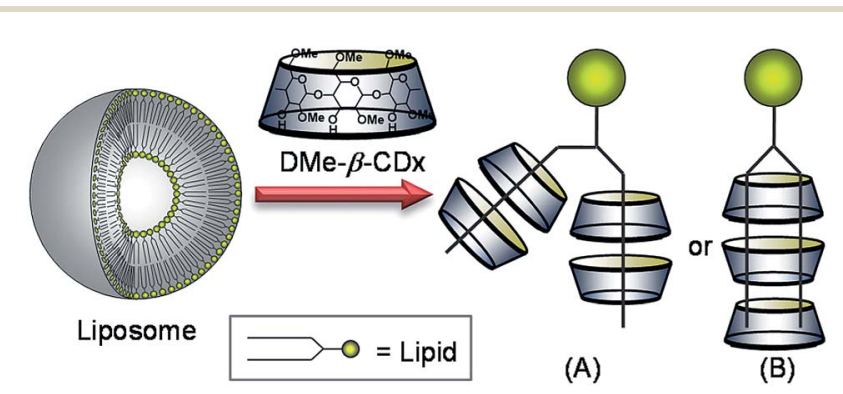

Scheme 1 Schematic illustration of the lipid-DMe- $\beta$-CDx complexes. 
the immobilization of its bowl shape conformation through a series of strong intramolecular hydrogen bonding interactions. Verrall et al. reported that DMe- $\beta$-CDx interacts with hydrocarbon surfactants much more effectively than TMe- $\beta$-CDx. ${ }^{21}$ Although these CDxs can include guest molecules through an induced-fit mechanism, the authors attributed the poor interaction efficiency of TMe- $\beta$-CDx compared with DMe- $\beta$-CDx to steric hindrance effects resulting from the increasing number of methyl groups. ${ }^{21}$

\section{Evaluation of the allosteric effects using Hill plots}

The proportion of isolated lipids relative to the total lipids $\left([\text { lipid }]_{\mathrm{c}} /[\text { lipid }]_{0}=y \text {; [lipid }\right]_{\mathrm{c}}$ : concentration of lipid in the lipid DMe- $\beta$-CDx, [lipid] $]_{0}$ : total concentration of lipid) can be readily estimated from the ratio of the peak intensities of the isolated lipid and DMe- $\beta$-CDx molecules in the ${ }^{1} \mathrm{H}$ NMR spectrum of a lipid-DMe- $\beta$-CDx complex (Fig. S5-S7†). A plot of the proportion of isolated DMPC versus [DMe- $\beta-\mathrm{CDx}]$ gave a sigmoidal curve, ${ }^{22-30}$ which indicated that the binding of DMe- $\beta$-CDx to DMPC occurred according to a well-defined cooperative phenomenon (Fig. 6A). The DMe- $\beta$-CDx-binding profile of this complex was analysed using the Hill equation: $\log [y /(1-y)]=$ $n \log [\mathrm{DMe}-\beta-\mathrm{CDx}]-\log K$, where $K$ and $n$ are the association constant and Hill coefficients, respectively, and $y=K /([$ guest $]-$ $n-K) .{ }^{31,32}$ In the range of $[\mathrm{DMe}-\beta-\mathrm{CDx}]=1.0-2.5 \mathrm{mM}(1.0-2.5$ equiv. for DMPC), the peak corresponding to the isolated DMPC molecules was too small to allow for the accurate calculation of $y$. From the slope and the intercept of the linear plot in the range of $[\mathrm{DMe}-\beta-\mathrm{CDx}]=5.0-20.0 \mathrm{mM}$, we obtained a $\log K$ value of $7.3\left(\Delta G=-41.6 \mathrm{~kJ} \mathrm{~mol}^{-1}\right)$ for the DMPC-DMe- $\beta$-CDx complex (correlation coefficient 0.992 ), together with an $n$ value of 3.6 (Fig. 6B). The fact that the Hill coefficient was close to 3.6 indicated that the complex would allow for the highly cooperative binding of three or four DMe- $\beta$-CDxs. The stepwise association constants for the DMPC-DMe- $\beta-C D x$ complex were subsequently estimated to be $\log K_{1}=0.8, \log K_{2}=2.2, \log K_{3}=$ 2.6 and $\log K_{4}=2.3$. It is noteworthy that the sum of these values was greater than 7.3 because of calculation errors. The predictions for these $K$ values were low because the interactions between DMe- $\beta-C D x$ and the alkyl chains of DMPC were inhibited by hydrophobic interactions between the alkyl chains of the DMPC molecules making up the liposomes.

The $\log K$ value for the DLPC-DMe- $\beta$-CDx complex was calculated to be $7.3\left(\Delta G=-41.6 \mathrm{~kJ} \mathrm{~mol}^{-1}\right)$ (correlation coefficient 0.981 ) with an $n$ value 3.0 (Fig. 6B). The Hill coefficient for the DLPC-DMe- $\beta$-CDx complex was therefore lower than that of the DMPC-DMe- $\beta$-CDx complex, which indicated that DLPC with its shorter alkyl chains could be dissolved by a complexation with a smaller number of DMe- $\beta$-CDxs in water. This result was therefore consistent with the observation that the DLPC liposomes collapsed following the addition of a much smaller amount of DMe- $\beta-\mathrm{CDx}([\mathrm{CDx}] /[\mathrm{DLPC}]=6$ equiv. $)$ compared with the DMPC liposomes (10 equiv.). The $\log K$ value was also calculated for the DPPC-DMe- $\beta$-CDx complex and found to be $6.3\left(\Delta G=-36.1 \mathrm{~kJ} \mathrm{~mol}^{-1}\right)$ (correlation coefficient 0.995$)$, with an $n$ value of 3.6 (Fig. 6B). Although the alkyl chains of DPPC are
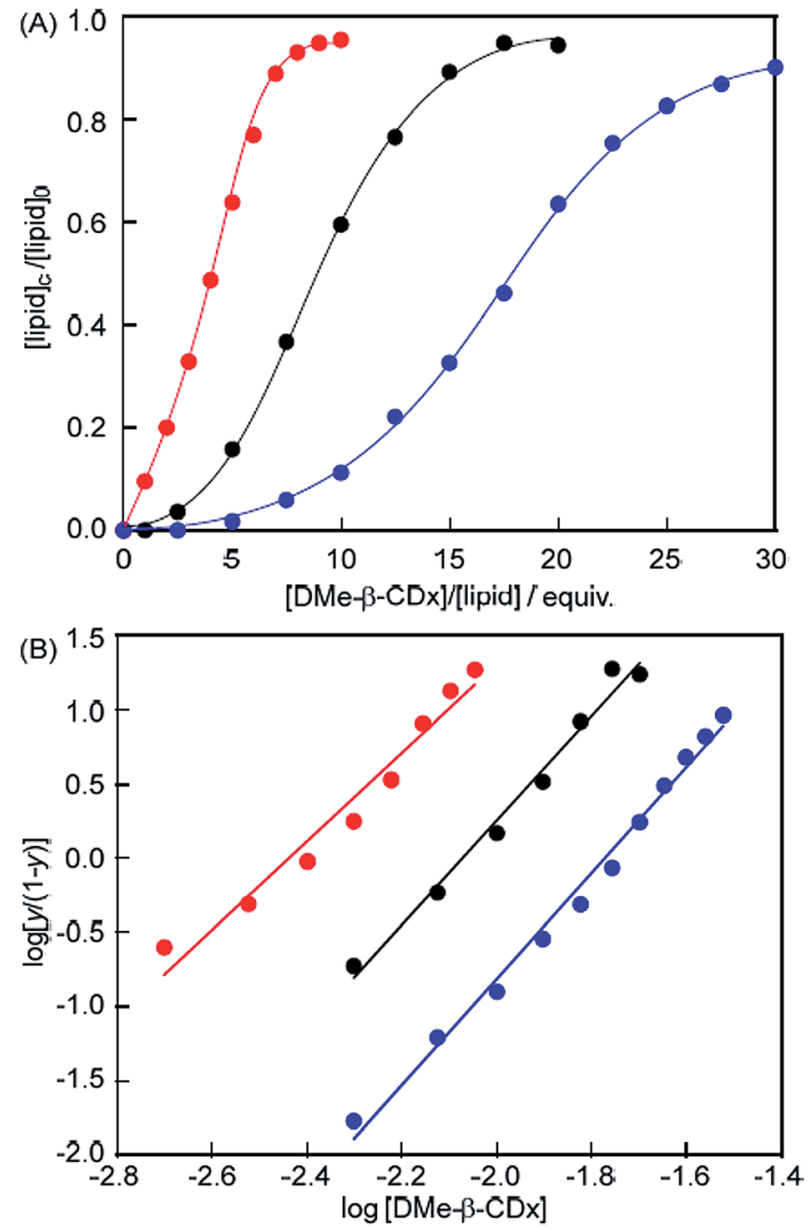

Fig. 6 (A) Plot of $[\text { lipid }]_{C} /[\text { lipid }]_{0}(=y)$ versus [DMe- $\left.\beta-C D x\right]$ \{llipid $]_{C}$ : concentration of lipid in the lipid-DMe- $\beta$-CDx complex, [lipid] $]_{0}$ : total concentration of lipid $(1.0 \mathrm{mM})\}$ and (B) plot of $\log [y /(1-y)]$ versus log [DMe- $\beta-C D x]$ for DMPC (black), DLPC (red) and DPPC (blue) liposomes.

longer than those of DMPC, the Hill coefficient for the DPPCDMe- $\beta$-CDx complex was similar to that of the DMPC-DMe- $\beta$ CDx complex. This result therefore indicated that the stability of the DPPC liposomes toward DMe- $\beta$-CDx could be attributed to the resulting complex having a lower association constant than the corresponding DMPC-DMe- $\beta$-CDx complex. The lower association constant of the DPPC-DMe- $\beta$-CDx complex could be attributed to differences in the interactions between the lipids in the liposomes during the initial states of the complexation, because there are very few differences in the nature of the interactions between the alkyl chains of DMPC and DPPC in the corresponding DMe- $\beta$-CDx complexes.

\section{Stoichiometry of lipid-CDx complexes}

The formation of interactions between multiple DMe- $\beta$-CDxs and a single lipid was further corroborated by cold-spray ionisation mass spectrometry (CSI-MS), which is a suitable technique for the detection of complexes held together by weak noncovalent interactions. All of CSI-MS spectra collected in the current study contained strong peaks with $\mathrm{m} / \mathrm{z}$ value of 1353.58 
and 1367.60, which indicated that the purchased DMe- $\beta$-CDx consisted not only of a compound that had been methylated at 14 of the hydroxyl groups on the 2- and 6-positions (1), but that it also contained a compound that had been methylated at 15 of the hydroxyl groups, including one at the 3-position (1-Me), as an impurity. The results of the CSI-MS analysis revealed that these compounds existed in a ratio of almost $3: 4$ based on the peak intensities. It is well known that it can be very difficult to separate and purify these compounds. ${ }^{33}$

The CSI-MS spectrum of the DMPC-DMe- $\beta$-CDx complex contained several strong peaks with $\mathrm{m} / \mathrm{z}$ values of 1016.54 for $[\mathrm{DMPC} \cdot \mathbf{1}+\mathrm{H}+\mathrm{Na}]^{2+}, 1689.34$ for $[\mathrm{DMPC} \cdot \mathbf{1} \cdot \mathbf{1}-\mathrm{Me}+\mathrm{H}+\mathrm{Na}]^{2+}$, 2362.13 for $[\mathrm{DMPC} \cdot \mathbf{1} \cdot \mathbf{2 1}-\mathrm{Me}+\mathrm{H}+\mathrm{Na}]^{2+}$ and 3027.91 for [DMPC $\cdot 21 \cdot 21-M e+\mathrm{H}+\mathrm{Na}]^{2+}$, which clearly indicated the presence of the DMPC-DMe- $\beta$-CDx complexes with molar ratios in the range of $1: 1-1: 4$ (Fig. 7). The CSI-MS spectra of the DLPC-DMe- $\beta$-CDx and DPPC-DMe- $\beta$-CDx complexes gave similarly strong peaks, which indicated that the corresponding lipid-DMe- $\beta$-CDx complexes were being formed with molar ratios in the range of $1: 1-1: 4$ (Fig. 8 and 9). Furthermore, the peaks corresponding to the $1: 4$ lipid-DMe- $\beta$-CDx complexes appeared as five different combinations between the $\mathbf{1}$ and 1-Me CDxs (Fig. 7B, 8B and 9B). The intensities of the peaks assignable to the lipid-DMe- $\beta$-CDx complexes decreased in the order of DPPC > DMPC > DLPC, which indicated that the ease with which these complexes were being formed was of the order DPPC > DMPC > DLPC. These results were therefore consistent
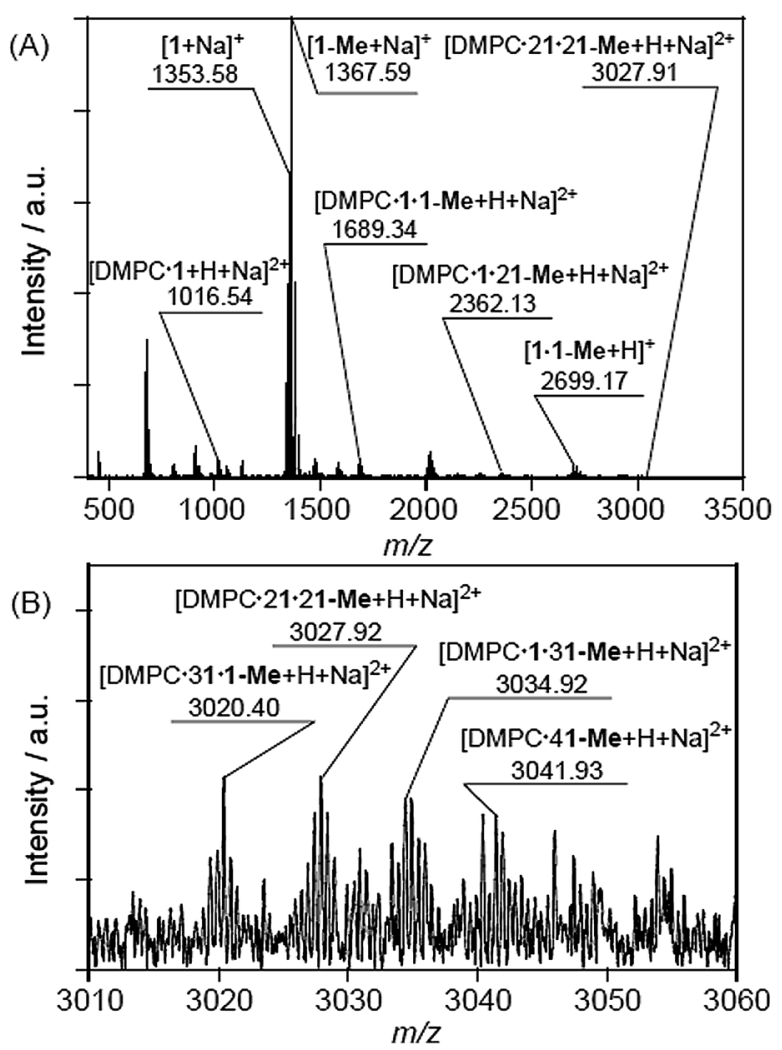

Fig. 7 (A) CSI-MS spectrum of DMPC-DMe- $\beta$-CDx complex and (B) expansion of the spectrum ([lipid] $=1.0 \mathrm{mM}$, [CDx]/[lipid] $=10$ equiv.).
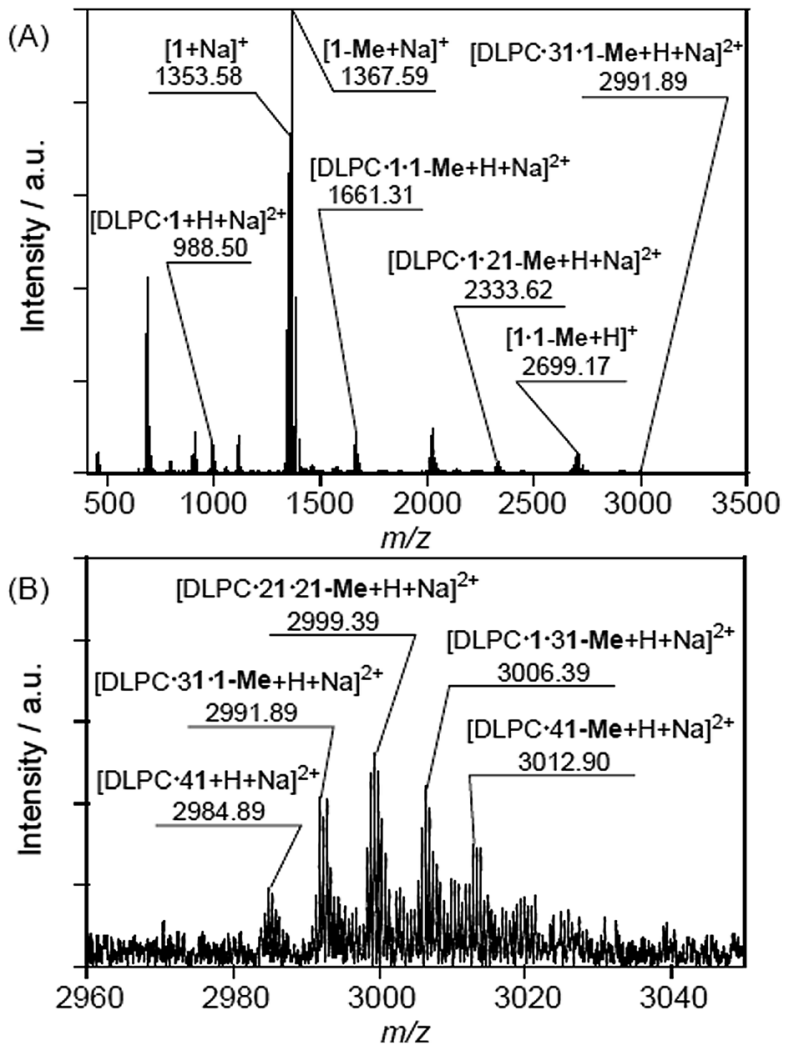

Fig. 8 (A) CSI-MS spectrum of DLPC-DMe- $\beta-C D x$ complex and (B) expansion of the spectrum ([lipid] $=1.0 \mathrm{mM},[C D x] /[$ lipid $]=10$ equiv.).
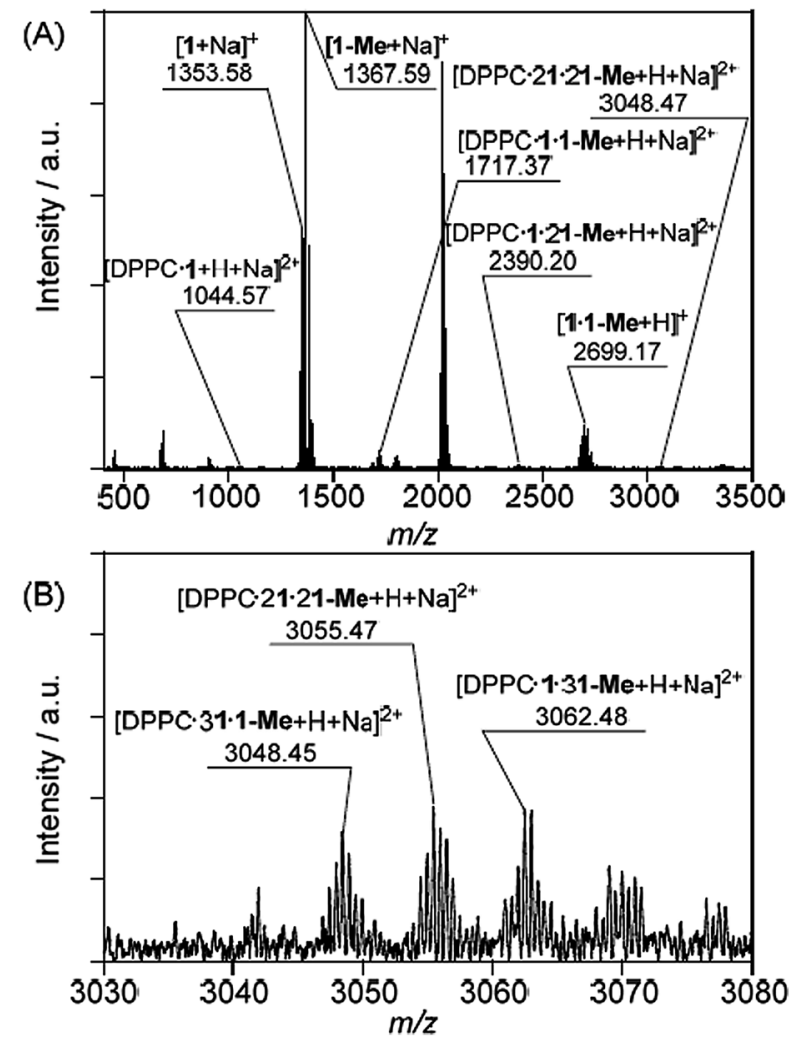

Fig. 9 (A) CSI-MS spectrum of DPPC-DMe- $\beta$-CDx complex and (B) expansion of the spectrum ([lipid] $=1.0 \mathrm{mM}$, [CDx]/[lipid] $=10$ equiv.). 
with those reported above for the intensity of the light scattering, DLS measurements and ${ }^{1} \mathrm{H}$ NMR analyses.

As shown in Scheme 1, there are two possible conformations for the lipid-DMe- $\beta$-CDx complexes including (i) two pairs of DMe- $\beta$-CDx molecules, with each CDx encapsulating a single alkyl chain (Scheme 1A) and (ii) several DMe- $\beta$-CDx molecules, with each CDx encapsulation two alkyl chains simultaneously. However, in explanation (ii), the alkyl chains would be too short to interact with three or four DMe- $\beta$-CDxs because alkyl chains $\left(\mathrm{C}_{11-15}\right)$ with an all anti conformation are approximately 12.7$17.8 \AA$ in length and the thickness of DMe- $\beta$-CDx is approximately $8 \AA$ A. The detection of $1: 3$ and $1: 4$ lipid-DMe- $\beta$-CDx complexes by CPI-MS therefore suggests that these complexes adopt a conformation similar to that provided by explanation (i).

\section{Effect of the addition of CDxs on plasma membranes}

As mentioned in the introduction, DMe- $\beta$-CDxs have been shown to exhibit high toxicity. ${ }^{7,8}$ However, these reports did not provide any evidence to suggest that DMe- $\beta$-CDxs brought about morphological changes in living cells. With this in mind, we examined the influence of the addition of CDxs on the plasma membranes of living cells. The introduction of CDxs to HeLa cells was observed by phase contrast microscopy (Fig. 10). After being treated with the DMe- $\beta$-CDx for $30 \mathrm{~min}$, almost all of the HeLa cells became spherical in shape (Fig. 10b). Although complete collapse of the cells was not observed, DMe- $\beta$-CDx gave damage to HeLa cells. In contrast, the plasma membranes of the HeLa cells changed significantly after being treated with TMe- $\beta$-CDx and $\gamma$-CD for $30 \mathrm{~min}$ (Fig. 10c and d). Arima et al. reported that the addition of TMe- $\beta$-CDx to cells resulted mainly in the release of cholesterol, whilst cholesterol and phospholipids were released from NR8383 cells. $^{34}$ The morphological
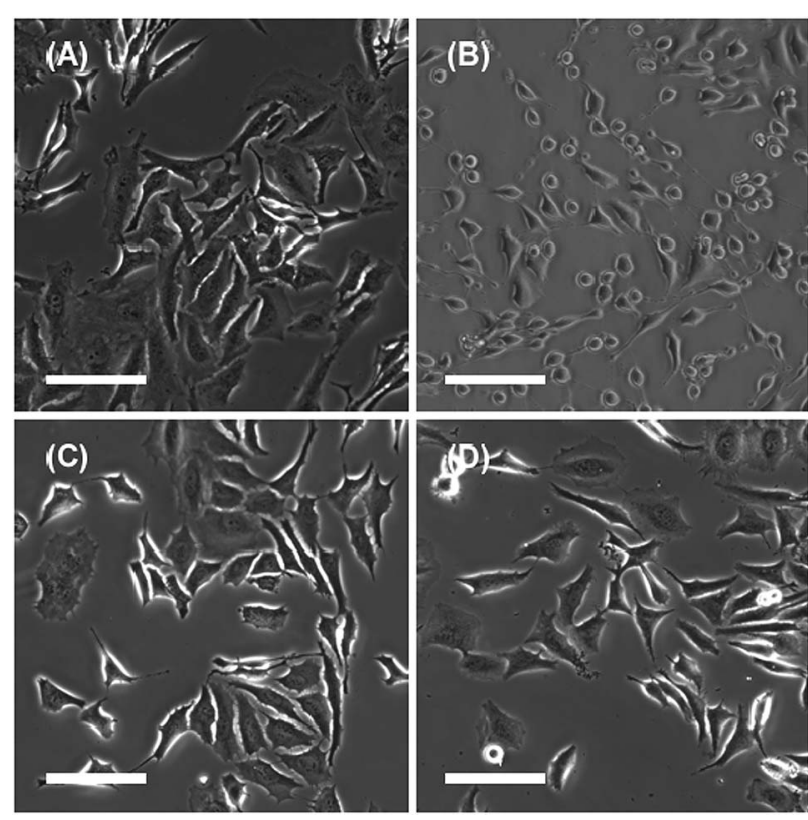

Fig. 10 Phase contrast images of the HeLa cells before $(A)$ and after being treated with (A) DMe- $\beta-C D x$, (B) TMe- $\beta-C D x$ and (C) $\gamma$-CDx for $30 \mathrm{~min}$ at $37^{\circ} \mathrm{C}$. The scale bar represents $100 \mu \mathrm{m}$ ([CDxs] $=10 \mathrm{mM}$ ). changes observed in the HeLa cells could therefore be attributed to the release of lipids and cholesterol from the plasma membrane in the presence of DMe- $\beta$-CDx.

\section{Experimental}

\section{Materials}

$\alpha$-CDx, $\beta$-CDx, $\gamma$-CDx, and TMe- $\beta$-CDx were purchased from Wako Pure Chemical Industries Ltd (Tokyo, Japan). DMe- $\beta$-CDx was purchased from Sigma-Aldrich Chemical Co., Inc. (St Louis, MO, USA). 1,2-Dimyristoyl-sn-glycero-3-phosphocholine (DMPC), 1,2-dilauroyl-sn-glycero-3-phosphocholine (DLPC), 1,2-dipalmitoyl-sn-glycero-3-phosphocholine (DPPC) and 1-palmitoyl-2-oleoyl-sn-glycero-3-phosphatidylcholine (POPC) were obtained from NOF Corp. (Tokyo, Japan).

\section{Preparation of liposomes}

Solutions of DMPC, DLPC and DPPC $\left(1.44 \times 10^{-5} \mathrm{~mol}\right)$ in chloroform $(1 \mathrm{~mL})$ were dried using a rotary evaporator at $40{ }^{\circ} \mathrm{C}$. Water $(4.0 \mathrm{~mL})$ was added to the solution, and the resulting mixture was shaken on a vortex mixer for $5 \mathrm{~min}$. To change from multilamellar to unilamellar vesicles and obtain a narrow size distribution, the solution was subjected to a freeze-thaw cycle (three times) and extruded eleven times (LiposoFast-Basic; Avestin Inc., Ottawa, Canada) with two stacked polycarbonate membranes, pore size $50 \mathrm{~nm}$. The resulting solution was diluted with water to a final concentration of $2.0 \mathrm{mM}$ lipids.

\section{Mixing of the liposomes with the cyclodextrins}

An aqueous solution of CDx (1.0 mL, 0-40.0 mM) was mixed with an aqueous solution of liposomes $(1.0 \mathrm{~mL}$, [lipid] $=$ $2.0 \mathrm{mM}$ ) at room temperature in a $5 \mathrm{~mL}$ glass vial. The $[\mathrm{CDx}] /[$ lipid] ratio was varied from 2.5 to 20 for DMPC, from 1.0 to 10 for DLPC and from 5.0 to 30 for DPPC.

\section{Light scattering measurement by the UV-vis absorption spectroscopy}

The UV-vis spectra were recorded using a UV-3600PC spectrophotometer (Shimadzu Corp., Kyoto, Japan). All of these experiments were performed at $25^{\circ} \mathrm{C}$ using a $1 \mathrm{~cm}$ cell.

\section{Dynamic light scattering (DLS)}

The hydrodynamic diameters of the liposomes were measured following the addition of the different CDx solutions using an electrophoretic light scattering instrument equipped with a laser Doppler system (Zetasizer Nano ZS, Malvern Instruments Ltd, Malvern, UK).

\section{Preparation of giant unilamellar vesicles (GUVs)}

GUVs were fabricated on indium tin oxide (ITO) electrodes using the electroformation method originally designed by Angelova et $a .^{35,36}$ This method provided facile access to a large amount of GUVs with diameters $>10 \mu \mathrm{m}$ under the appropriate conditions (see below). Ten microlitres of a lipid solution in chloroform with $1.0 \mathrm{mM}$ POPC was spread in a snakelike pattern without overlap 
over an area of $1.5 \times 1.5 \mathrm{~cm}^{2}$ using a $10 \mu \mathrm{L}$ Hamilton syringe. The lipid film deposited on the ITO-coated glass was then allowed to evaporate under a gentle stream of nitrogen gas. The electroformation chamber was then assembled. The chamber consisted of two ITO-coated coverslips with a copper wire, which were set up to be facing each other. The ITO-coated surfaces were separated by a $3 \mathrm{~mm}$ thick polydimethylsiloxane (PDMS) film, which was used to seal the chamber. The assembled vesicle electroformation chamber was slowly filled with $450 \mu \mathrm{L}$ of deionised water. A sinusoidal ac electric field of $10 \mathrm{~Hz}$ and $2.0 \mathrm{~V}$ (rms) was then applied to the chamber for $20 \mathrm{~min}$ to form GUVs at room temperature.

\section{${ }^{1}$ H NMR spectroscopy}

${ }^{1} \mathrm{H}$ NMR and $2 \mathrm{D}{ }^{1} \mathrm{H}^{1}{ }^{1} \mathrm{H}$ NOESY NMR spectra were recorded on Varian 400-NMR (400 MHz) (Varian Associates, Inc., Palo Alto, CA, USA) and JNM-ECA500 (500 MHz) spectrometers (JEOL Resonance Inc., Tokyo, Japan), respectively.

\section{Cold-spray ionisation mass spectrometry (CSI-MS)}

CSI-MS measurements were performed using a Fourier transform ion cyclotron resonance mass spectrometer (FT-ICR MS; Apex-Qe 9.4 T, Bruker Daltonics, Inc. Billerica, MA, USA). The heater for the desolvation assembly was turned off under the electrospray ionisation source and the temperature was held around $310 \mathrm{~K}$. The general CSI-MS conditions were as follows: positive ionisation mode; capillary voltage, $4.5 \mathrm{kV}$; dry gas flow rate, $5.0 \mathrm{~L} \mathrm{~min}{ }^{-1}$; nebulizer gas flow rate, $1.0 \mathrm{~L} \mathrm{~min}{ }^{-1}$; and sample flow rate, $120 \mu \mathrm{L} \mathrm{h}^{-1}$.

\section{Cell culture}

HeLa cells were maintained in $\mathrm{CO}_{2}$ independent medium (Gibco BRL) supplemented with $10 \%$ fetal calf serum at $37^{\circ} \mathrm{C}$ in $5 \% \mathrm{CO}_{2}$. The cells were seeded on glass-bottomed dishes at a density of $3.2 \times 10^{5}$ cells per plate. The cells were grown overnight under these conditions prior to be used in the experiments. Cells were seeded onto glass coverslips to allow for the uptake of the liposomes to be analysed.

\section{Fluorescence microscopy of HeLa cells}

The cells were pre-incubated at $37{ }^{\circ} \mathrm{C}$ without $\mathrm{CO}_{2}$ for $30 \mathrm{~min}$ before being treated with CDxs. Following a $30 \mathrm{~min}$ period of incubation with $10 \mathrm{mM}$ CDx complex at $37^{\circ} \mathrm{C}$, the cells were monitored by phase contrast microscopy. The cells were observed using an Olympus IX71 epifluorescence microscope equipped with a $200 \times$ objective lens. Phase contrast images were recorded using a Hamamatsu ImagEM EM-CCD camera (C9100-13).

\section{Conclusions}

In conclusion, the results of the current study have shown that liposomes collapse following the addition of a significant excess of DMe- $\beta$-CDx through allosteric interactions. Although similar phenomena have been observed following the addition of a suitable lysing agent or a surfactant under aqueous conditions, the lipid-DMe- $\beta$-CDx complexes generated in the current study were applicable to the Hill plots because the peaks of the lipids in the lipid-DMe- $\beta$-CDx complexes could be detected by ${ }^{1} \mathrm{H}$ NMR spectroscopy. The collapse of these liposomes was attributed on the formation of water soluble lipid-DMe- $\beta$-CDx complexes with stoichiometric ratios in range of $1: 1-1: 4$ through the construction of a pseudorotaxane structure, as shown by ${ }^{1} \mathrm{H}$ NMR and CSI-MS analyses. The stability of the liposomes evaluated in the current study towards DMe- $\beta$-CDx decreased in the order of DPPC $>$ DMPC $>$ DLPC, which correlated well with the length of the alkyl chains of the lipids. Furthermore, Hill plots for these complexes revealed that the interactions between the lipids and DMe- $\beta-C D x$ involved positive allosterism with Hill coefficient values in the range of 3.03.6. These results therefore suggest that the collapse of the liposomes results from them reaching a binding threshold in terms of the number of DMe- $\beta$-CDx molecules. Taken together, these observations provide an additional layer of information to explain why DMe- $\beta$-CDx is more cytotoxic than many other CDxs.

\section{Acknowledgements}

This work was supported by a JSPS KAKENHI a Grant-in-Aid for Scientific Research (B) (Grant No. 25288037).

\section{Notes and references}

1 J. Szejtli, Chem. Rev., 1998, 98, 1743-1753; G. Crini, Chem. Rev., 2014, 114, 10940-10975.

2 K. Uekama, J. Pharm. Soc. Jpn., 1981, 101, 857-873.

3 T. Loftsson and M. E. Brewster, J. Pharm. Sci., 1996, 85, 10171025.

4 K. Uekama, F. Hirayama and T. Irie, Chem. Rev., 1998, 98, 2045-2076.

5 T. Irie and K. Uekama, J. Pharm. Sci., 1997, 86, 147-162.

6 V. J. Stella and Q. He, Toxicol. Pathol., 2008, 36, 30-42.

7 F. W. H. M. Merkus, N. G. M. Schipper and J. C. Verhoef, J. Controlled Release, 1996, 41, 69-75.

8 S. Gould and R. Scott, Food Chem. Toxicol., 2005, 43, 14511459.

9 S. K. Rodal, G. Skretting, O. Garred, F. Vilhardt, B. van Deurs and K. Sandvig, Mol. Biol. Cell, 1999, 10, 961-974.

10 D. Castagne, G. Dive, B. Evrard, M. Frédérich and G. Piel, J. Pharm. Pharm. Sci., 2010, 13, 362-377.

11 J. Szejtli, T. Cserhati and M. Szogyi, Carbohydr. Polym., 1986, 6, 35-49.

12 J. Nishijo and H. Mizuno, Chem. Pharm. Bull., 1998, 46, 120124.

13 G. Piel, M. Piette, V. Barillaro, D. Castagne, B. Evrard and L. Delattre, Int. J. Pharm., 2007, 338, 35-42.

14 S. M. K. Davidson and S. L. Regen, Chem. Rev., 1997, 97, 1269-1279 and references therein.

15 A. Mueller and D. F. O'Brien, Chem. Rev., 2002, 102, 727-757 and references therein.

16 I. Puskás and F. Csempesz, Colloids Surf., B, 2007, 58, 218224. 
$17 \beta$-CDx was too low water-solubility to measure an intensity of light scattering at $450 \mathrm{~nm}$ by a UV-vis absorption spectra in the same conditions.

18 Delay times until a shrink of the GUV were unsettled because a sample had a distance between the GUV and the tip of micropipette.

19 A. Harada, J. Li and M. Kamachi, Chem. Lett., 1993, 237-240.

20 A. Harada, J. Li, S. Suzuki and M. Kamachi, Macromolecules, 1993, 26, 5267-5268.

21 L. D. Wilson and R. E. Verrall, Can. J. Chem., 1998, 76, 25-34.

22 S. Shinkai, A. Sugasaki, M. Ikeda and M. Takeuchi, Acc. Chem. Res., 2001, 34, 494-503.

23 M. Takeuchi, A. Sugasaki, M. Ikeda and S. Shinkai, Acc. Chem. Res., 2001, 34, 865-873.

24 M. Takeuchi, T. Imada and S. Shinkai, Angew. Chem., Int. Ed., 1998, 37, 2096-2099.

25 A. Sugasaki, M. Ikeda, M. Takeuchi and S. Shinkai, Angew. Chem., Int. Ed., 2000, 39, 3839-3842.

26 A. Sugasaki, K. Sugiyasu, M. Ikeda, M. Takeuchi and S. Shinkai, J. Am. Chem. Soc., 2001, 123, 10239-10244.
27 O. Hirata, M. Takeuchi and S. Shinkai, Chem. Commun., 2005, 3805-3807.

28 T. Ikeda, O. Hirata, M. Takeuchi and S. Shinkai, J. Am. Chem. Soc., 2006, 128, 16008-16009.

29 M. Ikeda, Y. Kubo, K. Yamashita, T. Ikeda, M. Takeuchi and S. Shinkai, Eur. J. Org. Chem., 2007, 2007, 1883-1886.

30 R. Wakabayashi, T. Ikeda, Y. Kubo, S. Shinkai and M. Takeuchi, Angew. Chem., Int. Ed., 2009, 48, 6667-6670.

31 B. Perlmutter-Hayman, Acc. Chem. Res., 1986, 19, 90-96.

32 K. A. Connors, Binding Constants, Wiley, New York, 1987.

33 C. M. Spencer, J. F. Stoddart and R. Zarzycki, J. Chem. Soc., Perkin Trans. 2, 1987, 1323-1335.

34 K. Motoyama, K. Kameyama, R. Onodera, N. Araki, F. Hirayama, K. Uekama and H. Arima, Eur. J. Pharm. Sci., 2009, 38, 249-261.

35 M. Angelova and D. Dimitrov, Faraday Discuss. Chem. Soc., 1986, 81, 303-311.

36 G. Staneva, M. I. Angelova and K. Koumanov, Chem. Phys. Lipids, 2004, 129, 53-62. 